\section{$\underset{\substack{\text { hommes } \\ \text { \& migrations }}}{ }$}

\section{Hommes \& migrations}

Revue française de référence sur les dynamiques

migratoires

\section{$1308 \mid 2014$}

Les Paris des migrants

\title{
Travailleuses sans-papiers à Paris
}

Retour sur la tentative de constitution d'un collectif de femmes

Joanne Le Bars

\section{OpenEdition}

1 Journals

\section{Édition électronique}

URL : http://journals.openedition.org/hommesmigrations/3006

DOI : 10.4000/hommesmigrations.3006

ISSN : 2262-3353

Éditeur

Musée national de l'histoire de l'immigration

\section{Édition imprimée}

Date de publication : 1 octobre 2014

Pagination : 105-111

ISBN : 978-2-919040-29-2

ISSN : 1142-852X

\section{Référence électronique}

Joanne Le Bars, "Travailleuses sans-papiers à Paris », Hommes \& migrations [En ligne], 1308 | 2014, mis en ligne le 01 octobre 2017, consulté le 30 avril 2019. URL : http://journals.openedition.org/

hommesmigrations/3006; DOI : 10.4000/hommesmigrations.3006 


\title{
TRAVAILLEUSES \\ SANS-PAPIERS À PARIS RETOUR SUR LA TENTATIVE DE CONSTITUTION D'UN COLLECTIF DE FEMMES
}

par JOANNE LE BARS, géographe, Lab'Urba, allocataire Institut Émilie du Châtelet.

\author{
Confrontées à des discriminations croisées dues à leur sexe \\ et à leur origine ethnique, à Paris, les travailleuses migrantes \\ commencent à faire entendre leur voix dans les mobilisations \\ de sans-papiers. Suite à I'occupation d'un lieu parisien, \\ l'émergence d'un collectif de femmes sans-papiers va mettre \\ en avant leur expérience racialisée de travailleuses \\ domestiques. Leur souhait de manifester dans I'espace public \\ traduit leur volonté d'accéder à une reconnaissance sociale.
}

Paris a vu l'émergence des mouvements de sanspapiers. Alors que de nombreuses recherches se sont intéressées aux mouvements sociaux, notamment ceux concernant des sans-papiers ${ }^{1}$, peu d'entre elles se sont expressément penchées sur les femmes sans-papiers dans les mobilisations collectives, et moins encore aux croisements des catégories de sexe, de "race" et de classe, dans l'analyse de ces mouvements et de l'expérience de ses actrices². Revenant à mes premiers pas ethnographiques avec des femmes sans-papiers au sein d'un lieu d'occupation parisien, j'ai souhaité me focaliser dans cet article sur la difficile construction d'un collectif de femmes sans-papiers, à travers l'analyse des positions de trois habitantes de ce lieu, que je nommerai Fatoumata, Bintou et Lela, toutes trois arrivées de Bamako dans les années $2000^{3}$.

Le projet de constituer un groupe de femmes, rendant centrales la question de leur travail et leur volonté de manifester dans l'espace public, participe au questionnement de l'image sociale d'un groupe rendu illégitime, celui de "femmes sans-papiers". Dans ce contexte, les propos des femmes soulignent la continuité complexe et sans cesse redéfi- 
nie des politiques d'un État postcolonial néolibéral dans la construction de corps travailleurs, notamment ceux des travailleuses domestiques racialisées ${ }^{4}$. Les lois et politiques migratoires construisent la catégorie des "sans-papiers", constituant une main-d'œuvre précarisée, docile et servile ${ }^{5}$, et participent au confinement des femmes immigrées, notamment celles nouvellement arrivées, à un travail domestique au noir ${ }^{6}$. La volonté d'arborer des tee-shirts rouges dans la

En tant que femmes sans-papiers, leur rapport à la ville combine plusieurs dimensions, depuis les expériences de la domination au travail jusqu'à la résistance revendiquée, depuis l'occupation de lieux militants jusqu'à la visibilité dans les espaces publics. rue et devant une mairie va à l'encontre des processus d'invisibilisation qui marquent leur pratique quotidienne.

En filigrane, le retour sur cette expérience nous offre un regard sur leurs expériences citadines à Paris. En tant que femmes sans-papiers, leur rapport à la ville combine plusieurs dimensions, depuis les expériences de la domination au travail jusquà la résistance revendiquée, depuis l'occupation de lieux militants jusqu’à la visibilité dans les espaces publics.

\section{D'une posture militante à une démarche de recherche}

En décembre 2009, je me rendais pour la première fois dans un lieu d'occupation à Paris. Ma venue était liée à la mise en place d'ateliers de vidéo dits "participatifs" avec des femmes sans-papiers. Ce n'est que par la suite que ce lieu deviendra mon terrain de master-2 et me conduira à ma thèse de géographie. Débute alors une enquête ethnographique auprès de femmes sans-papiers dans la région parisienne. Cette enquête s'est déroulée au sein de ce lieu d'occupation, d'une association dite de "défense des sans-papiers", de lieux d'hébergement et d'accueil du 115, d'une association de "santé communautaire" et enfin de deux PMI (Centres de protection maternelle et infantile). Ces lieux sont liés par un réseau d'interconnaissance, du moins par des relations d'interdépendance. Des entretiens ont été effectués avec des professionnels du corps médical, psycho-social et associatif. Des entretiens formels et informels avec 70 femmes dites "sans-papiers" originaires d'Afrique subsaharienne et du Maghreb ont pu être faits. La rencontre avec Fatoumata, Bintou et Lela, dont les récits constituent le matériel de cet article, date de janvier 2010 dans ce lieu d'occupation. Ces trois femmes âgées de 40 à 50 ans partagent la même chambre. Des liens forts les unissent. Depuis quatre ans, je les ai rencontrées à de nombreuses reprises. Fatoumata et Bintou sont devenues des interlocutrices privilégiées. $\mathrm{Au}$ sein de ce lieu d'occupation, la signification de ma présence a été constamment négociée. Le rôle que l'on m'a attribué oscillait en fonction du contexte et de l'avancée de l'enquête entre celui de journaliste, celui de militante et celui d'étudiante. J'étais également renvoyée à mon appartenance à une société postcoloniale, à ma supposée religion catholique, à ma nationalité, à ma couleur de peau ou à mon statut de femme non mariée sans enfants, etc.

\section{Les femmes dans les mouvements collectifs de sans-papiers}

C'est tardivement que la figure du travailleur et plus encore celle de la travailleuse sans-papiers arrivent dans les revendications et se traduisent par différents modes d'action. L'histoire des mouvements de sans-papiers à Paris commence dans les années 1970 et se construit au fur et à mesure 


\section{QUELQUES DATES}

/// 1973: 52 ouvriers de la société Margoline se mettent en grève, à Nanterre et à Gennevilliers.

\section{/// 1980 : Grève des Turcs} sans-papiers parisiens du Sentier (Paris II).

/// 1996 : Occupation de l'église Saint-Ambroise, du gymnase Japy, des locaux syndicaux de Sud-PTT, de la Cartoucherie de Vincennes, des entrepôts SNCF rue Pajol, pour arriver à l'occupation de l'église Saint-Bernard.

/// 2002 : Occupation de la basilique de Saint-Denis.

/// 2004 : Marche européenne des sans-papiers de Bruxelles à Paris.
/// 2006 : Première vague de grèves lancées par la CGT. Grève à la blanchisserie Modeluxe.

/// 2007 : Grèves dans différentes entreprises sur quelques sites de la région parisienne.

/// 2008 : Grèves dans différentes entreprises de la région parisienne, dont celle des travailleuses de MaNet, puis de DMMS (dont l'activité principale est le nettoyage). Occupation de l'église Saint-Paul à Nanterre et de la Bourse du travail à Paris.

/// 2009 : Occupation de la CPAM (Paris XVIIIe).

/// 2010 : Marche de Paris à Nice à l'occasion de la commémoration du $50^{\mathrm{e}}$ anniversaire de l'indépendance des pays

africains ; occupation

de la Cité nationale de l'histoire de l'immigration.

/// 2011 : La Caravane des sanspapiers, Forum social mondial à Dakar.

/// 2012 : Marche européenne des sans-papiers et migrant-e-s (Belgique, France, Allemagne, Suisse, Italie).

/// 2012-2013: Grévistes de la faim sans-papiers à Lille.

/// 2013 : La Caravane des sanspapiers, Forum social mondial à Tunis.

/// 2013: Marche du Grand Paris des sans-papiers. des tentatives et des expérimentations de formes de résistance collective (voir encadré). La notion de "sans-papiers" se construit dans l'espace public à partir de 1972-1973, au moment des circulaires Marcellin-Fontanet ${ }^{7}$. De la revendication d'appartenance à une commune humanité dans les premiers modes d'action des sans-papiers, on passe progressivement à la figure du "travailleur sanspapiers" et de la "travailleuse sans-papiers" à partir des grèves de 2007-2008 ${ }^{8}$. Les lieux et modes d'action changent : les grèves et l'occupation d'entreprises sont davantage privilégiées. En 1996, l'église Saint-Bernard (XVIII arr.) et l'église SaintAmbroise ( $\mathrm{XI}^{\mathrm{e}}$ arr.) sont occupées ; en 2008, les vagues de grèves et occupations se déroulent dans des entreprises. Certaines ont lieu dans des lieux centraux, comme par exemple les 5 restaurants de l'avenue des Champs-Élysées occupés le 20 mai 2008. L'avenue est rebaptisée "l'avenue des champs-papiers".

L'apparition du terme "travailleur" ne fait pas l'unanimité et génère un clivage au sein des mouvements de sans-papiers, certains réclamant la régularisation de "tous les sans-papiers ${ }^{10}$ ". C'est dans ce contexte qu'émerge le collectif où j'ai mené mon enquête.

Au moment où je les rencontre, en 2009, Lela et Fatoumata ont déjà une expérience d'occupation. Leurs trajectoires militantes nous rappellent que les femmes immigrées, notamment sans-papiers, 
ont constitué de nombreux mouvements collectifs dont il a été peu fait état ; elles ont également participé à des mobilisations et ce depuis au moins les années 1970 en France ${ }^{11}$. Ces mouvements divers se trouvent au croisement du mouvement pour les droits des migrants et du mouvement féministe ${ }^{12}$. Plus spécifiquement, dans les collectifs de sanspapiers, les femmes ont joué un rôle important $t^{13}$. Des revendications spécifiques concernant la situation des femmes sans-papiers et le travail qu'elles effectuent émergent. Après les mouvements de grèves et de revendications de 2007-2008, soutenus par des associations et des syndicats, des femmes ont obtenu un titre de séjour mais "au prix d'une assignation à un métier conçu comme féminin $n^{14 "}$.

\section{Un lieu d'occupation parisien}

Un vaste bâtiment. Deux immenses banderoles sont accrochées sur la façade : "Régularisation de tous les sans-papiers" et "Fermeture des centres de rétention". Il faut passer sous un grand porche gardé par quelques hommes, se présenter aux "vigiles" (comme les appellent les femmes rencontrées) lorsqu'on n'est pas des lieux, pour arriver dans une vaste cour. Lété, une télé y est installée, des personnes sont assises devant, en majorité des hommes. Des femmes préparent le repas distribué une fois par jour, entre 13 h 30-14 heures. L'hiver, il y a moins de monde. Les cuisinières sont dans un recoin, à l'abri du vent. Arrivée dans la cour, je tourne à gauche sous un autre porche et monte jusqu'au deuxième étage, étage des femmes. Une vaste pièce a été transformée en salle de prière. Certaines femmes s'y rendent, d'autres prient dans leur chambre. Une manifestation des sans-papiers part de ce lieu tous les mercredis. Dans la chambre où logent les femmes rencontrées, des lits doubles sont posés sur le sol.

Pour la majorité des femmes que j’ai connues dans ce lieu, la raison pour laquelle elles sont venues s'y installer, c'est qu'elles n'avaient pas de logement. L'autre alternative était le 115 (hébergements d'urgence du Samu social), dont l'expérience a été éprouvante. Bintou a appris l'occupation du lieu par un ami. Arrivée à Paris en 2001, elle est accueillie par une femme qu'elle connaît du Mali. Cette femme l'hébergeait contre une participation financière. Sans revenus, elle part s'installer dans un premier lieu d'occupation : "elle [son hébergeante] est gentille quand tu as de l'argent, mais quand tu n'as plus d'argent...", me dira-t-elle. Fatoumata rejoint en 2008 la Bourse du travail occupée (à proximité de la place de la République), mais elle a recours conjointement au 115 en raison des conditions difficiles au sein du lieu occupé : "Je suis restée à la République au moins pendant quelques moments et je pouvais faire deux ou trois jours sans me laver, ça me gênait. Je les ai appelés, j'ai dit: 'Madame, je veux que vous me mettiez dans un lieu quel qu'en soit le lieu." En 2009, elle poursuit avec une autre occupation, où je la rencontre. Elle y habitera jusqu'en août 2010.

Dans les années qui ont suivi, les femmes évoquent leurs souvenirs de cette expérience des lieux occupés. "On écoutait de la musique malienne", dit Bintou. Les douches étaient parfois prises dans les toilettes du premier étage. L'eau était chauffée à la bouilloire électrique. D'autres se rendaient aux douches publiques. Le linge était lavé à la main dans des seaux ou parfois amené au pressing lorsqu'elles avaient assez d'argent. Leurs affaires étaient conservées dans différents endroits. Fatoumata, par exemple, avait des bagages dans cinq endroits : "Chez des Blancs, des Noirs, en banlieue et à Paris. Mon souhait était de rassembler tout ça et de pouvoir y accéder quand je veux". Elle a donc décidé avec d'autres personnes de louer un box de rangement dans le Nord-Est parisien pour 130 euros par mois. Il fait $4 \mathrm{~m}^{2}$, "C'est ma maison!" s'exclame-t-elle. 


\section{Créer un collectif de femmes sans-papiers}

En juin 2010, suite à une réunion avec les délégués du collectif, Fatoumata est assise dans sa chambre avec Bintou. Elle m'explique qu'elle a décidé de "convoquer d'elle-même une réunion entre les femmes du squat et les soutiens des femmes". Elles veulent prendre l'initiative de "rencontrer le préfet et lui dire qu'elles travaillent ici et cotisent". Bintou termine la conversation par un "c'est l'émancipation". Quelques mois après l'évacuation des lieux, Fatoumata m'a sollicitée pour soutenir leur groupe d'anciennes habitantes du lieu, pour les aider à créer un site Internet effectivement dédié aux femmes. "Et avec ce site, on veut faire une communication avec la Préfecture et la mairie."

Première réunion à Paris en 2011. Quinze femmes sont assises autour d'une vaste table. Fatoumata prend la parole: "Moi je suis votre griotte. Je ne sais pas bien parler, je suis pas allée loin, mais si on décide de parler dans notre langue bambara, on va parler et quelqu'un va traduire. Il faut qu'on se donne la main, il faut qu'on parle. Pas de peur, on laisse nos peurs à côté." Des femmes arrivent par intermittence dans la pièce, d'autres appellent Fatoumata sur son téléphone pour lui demander le chemin, entrecoupant ses propos. Elle insiste sur le nécessaire engagement collectif lors de la réunion: "C'est l'union qui fait la force, si on est nombreux, on peut faire quelque chose, nous les femmes. Si on est nombreuses, on se trahit pas, on se donne la main, on peut faire quelque chose."

Ces femmes ont choisi de nous associer, une de mes amies et moi, mais de manière secondaire, en tant que soutien "technique", parce que, au-delà du clivage qu'elles ont relevé à l'intérieur du mouvement des sans-papiers - soulignant l'existence de relations de pouvoir entre "soutiens" (pas sanspapiers) et sans-papiers -, elles disaient de nous :
"Ce sont des enfants, mais ce qu'ils connaissent, nous on ne connait pas. (...) Ils sont dans leur pays. Ils connaissent ce qui se passe ici sur le terrain, donc on se confie à eux."

\section{"Pour eux, on est juste des COD, des compléments d'objet direct"}

Ce qui ressort des échanges au sein de leur collectif, c'est avant tout leur expérience de travailleuses. Les seuls emplois qui leur soient ouverts dans l'agglomération parisienne concernent le nettoyage, l'entretien ou l'aide à domicile. Ces emplois témoignent d'une division raciale du travail. "On veut faire sortir nos voix. Nous les femmes, nous sommes là (...). Nous sommes dans les ménages, nous sommes dans les gardes d'enfants.

Les Blancs ont besoin de nous ce qui ressort des échanges nuit et jour. Nous sommes les au sein de leur collectif, premières à prendre le métro c'est avant tout leur expérience à 5 heures du matin. C'est de travailleuses. Les seuls nous qui sommes là à nous occuper de leurs bureaux, de leurs enfants." Fatoumata, lors d'une réunion, nous appelle à revenir sur leurs emplois qui leur soient ouverts dans l'agglomération parisienne concernent le nettoyage, l'entretien ou l'aide à domicile. expériences racialisées de travailleuses domestiques dans le secteur informel. Depuis que je la connais, Fatoumata garde des jumeaux dans la même famille. Dans le contexte des entretiens individuels, ou lors de réunions, elle s'attache à souligner le "sale boulot ${ }^{15}$ " qu'elle a à effectuer: "La première fois que j'ai commencé le ménage, ça a commencé à enlever la peau de ma main, c'est enflé, ça a pris de l'eau, après y a la peau qui est partie, je me suis arrêtée" (entretien, juillet 2011).

Lela, assise surson lit, évoque son travail denettoyage dans les gares de la région parisienne: "Il y a des gens qui vomissent, il y en a même qui font caca. Le matin, je ramasse ça" (août 2010, carnet de terrain), un travail 
invisible effectué par les nettoyeur(ses) de train ${ }^{16}$. Un jour de juillet 2010, au cours d'une de mes nombreuses visites, Lela me dit: "Ça fait longtemps que je n'ai pas déclenché l'alarme au travail." Il lui arrive très rarement d'utiliser ce moyen afin de finir plus tôt lorsqu'elle est trop épuisée. Cet acte fait écho aux actes de résistance et de révolte souterraines décrits par Nicolas Jounin ${ }^{17}$ en ce qui concerne les travailleurs du bâtiment en situation de vulnérabilité sociale et juridique.

Comme dans la plupart des secteurs subalternes, les lieux de travail sont dissociés, voire très éloignés les uns des autres, obligeant les femmes à des déplacements quotidiens contraignants. "Nous, on se déplace beaucoup, on bouge beaucoup, c'est pourquoi on connaît mieux Paris", me dit Lela. Dans une même journée, elle fait le nettoyage dans différentes gares du Nord-Ouest de la région parisienne : Épluches, Pontoise, puis Saint-Ouen-l'Aumône-Quartierde-l'Église. Elle travaille de 6 à 12 heures puis de 15 à 18 heures pour 800 euros par mois. Elle me raconte qu'au Mali, on dit que "les Maliens qui vivent en France ont les mains sèches, ce sont les mains du ménage" (août 2010, carnet de terrain).

Certaines recherches sur le travail domestique ont mis en valeur la dimension "relationnelle" et affective du travail domestique. Mais ces femmes ne se situent pas seulement au niveau des relations interindividuelles avec leurs employeurs, elles appréhendent aussi les rapports de domination liés au travail domestique ${ }^{18}$.

C'est la dimension "sale boulot" de la reproduction sociale "plus proche de la servilité que de la sublimation morale ${ }^{19 "}$ qui s'impose, comme l'ont montré Nasima Moujoud et Jules Falquet ou encore Rose-Myrlie Joseph ${ }^{20}$. Ces récits soulignent également le fait que ce travail n'est pas un "choix" et n'est pas effectué par n'importe qui ${ }^{21}$, comme le dit
Lela (août 2010) : "La femme noire n'est pas considérée (...), pour eux, on est juste des COD, des compléments d'objet direct." Fatoumata et Lela évoquent leur force de travail et leur exploitation en tant que femmes sans-papiers et perçoivent "la sexuation des mécanismes de l'exploitation 22". Ce qui permet d'éviter l'émergence de constructions essentialisantes, sexistes et racistes postulant que ce travail serait "naturellement" féminin ou que les "migrantes [auraient] une aptitude culturelle pour ce travail23".

Ces femmes, dont certaines sont qualifiées, effectuent des travaux domestiques non protégés, à temps partiel, payés au noir et au rabais, dont les lieux et horaires sont dispersés dans le temps et l'espace. Ces contraintes contribuent à les isoler et représentent un frein à leur syndicalisation. Cela rappelle la précarisation légale de ces femmes et "limportance historique du travail de reproduction sociale subi par des femmes étrangères ou racialisées ${ }^{24 "}$.

\section{Passer inaperçu dans l'espace public parisien}

Le rapport des femmes sans-papiers à l'espace public est habituellement marqué par l'invisibilité. Certes, dans les entretiens, elles précisent toutes qu'elles n'ont pas peur d'être dans l'espace public parisien, ni de croiser des policiers. Lors d'un entretien avec Fatoumata (2011), à ma question "Et tu n'as jamais eu peur des policiers?", elle répond: "Jamais, jamais. J'ai eu peur seulement de sortir sans le ticket. Depuis que je suis ici, ma cousine, elle me dit qu'en France, les policiers ne contrôlent jamais une femme sauf si tu passes derrière quelqu'un, que tu n'as pas de ticket, là ils te pardonnent pas. Arrêter une femme, tant que tu n'es pas soupçonnée de quelque chose, jamais. Depuis qu'on m'a 
dit ça, c'est dans ma tête, donc je n'ai pas peur. Parfois quand ils viennent, je me dirige vers les policiers même." Il conviendrait d'observer ce décalage entre les femmes et les hommes sans papiers en ce qui concerne la possibilité d'être arrêté. En revanche, dans des contextes informels, elles racontent l'ensemble des stratégies qu'elles adoptent pour tenter de passer inaperçues. Cela concerne la mise en scène du corps, sa posture, l'habillement, les jeux de regards et l'évitement de certains lieux. Une femme me racontait qu'un jour où elle rentrait tard chez elle en métro, elle aperçoit des policiers à la gare Saint-Lazare. Elle me dit : "Heureusement, jétais bien habillée, jeétais classe." Puis elle ajoute: "Comme jétais avec un tailleur, il m'a même pas regardée" (carnet de terrain, 2010). D'autres ont évoqué le fait qu'elles paraissaient "blanches", ce qui leur évitait d'être contrôlées. Ces arguments mettent en exergue les normes traversant l'espace public avec l'existence de féminités "socialement acceptables". Stéphane Le Courant évoque le "gouvernement par la peur" et une "intériorisation de la menace ${ }^{25 ",}$ afin d"insécuriser ceux qui restent sur le territoire". Ces stratégies mettent aussi en lumière les processus construisant ces femmes comme corps "hors normes", les rendant "space invaders", pour reprendre les termes de Puwar ${ }^{26}$.

\section{Un combat pour la visibilité}

"Rouge! Tout, tout rouge! On va se retrouver devant la mairie, toute la journée, devant la mairie ${ }^{27}$." Paris, avec ses avenues, ses places et son espace public, est un lieu d'action pour les revendications. La volonté de se "rendre visible" a occupé une place importante au sein des discussions entre les femmes. Porter des tee-shirts de couleur devant la mairie, qu'elles identifient comme une institution représentative de l'État à l'échelle locale (celle de l'arrondissement où se situe leur lieu d'occupation), permet d'inscrire dans l'espace ${ }^{28}$ la revendication des femmes "sans-papiers". "Se faire plus voir", comme l'expliquait Fatoumata (entretien individuel, 2011), soulève au moins deux enjeux ayant trait à la reconnaissance et aux régimes de visibilité dans l'espace urbain qui touchent de façon particulière les sanspapiers, comme l'a montré Clara Lecadet à propos des grévistes sans papiers et des migrants expulsés vers le Malii ${ }^{29}$.

La volonté de se mettre en scène collectivement dans l'espace public trouble l'ordre spatial dominant. Le choix de la visibilisation éclaire les processus de construction des normes de "race", de genre, de sexualités et de classe qui traversent l'espace public. Les manifestations des femmes sanspapiers qui ont eu lieu dans les rues de Paris et des communes proches ont donc une importance capitale pour comprendre la capacité de ces femmes à subvertir l'ordre spatial et social dominant.

En ce sens, mettre en scène collectivement son corps de travailleuse sans papiers en arborant un tee-shirt rouge apparaît comme un principe de distinction qui tranche avec les régimes de visibilité et de "placement" des corps. Par ce mouvement, elles rejettent le positionnement normatif périphérique qui leur est assigné "symboliquement et géographiquement $t^{30}$. Le changement de signification engendré par cet acte contestataire contient un potentiel subversif et concrétise l'apparition de nouvelles actrices.

25. Stéphane Le Courant, "L'intériorisation de la frontière sous menace d'expulsion. Le quotidien des étrangers en situation irrégulière", in Didier Fassin (dir.), Les Nouvelles Frontières de la société française, Paris, La Découverte, 2010, pp. 455-476. 26. Nirmal Puwar, Space Invaders. Race, Gender and Bodies Out of Place, Berg Publishers, 2004. 27. Fatoumata, réunion collective, 2011. 28. Fabrice Ripoll, “L'appropriation de l'espace au regard des mouvements sociaux contemporains : quelques réflexions sur les enjeux, modalités et ressources de l'action”, in Travaux et documents de l'UMR 6590, n²1, 2004, pp. 45-50. 29. Clara Lecadet, "Grévistes sans-papiers et migrants expulsés vers le Mali : dialectique du visible et de l'invisible dans des formes inédites de luttes sociales”, op. cit. 30. Marianne Blidon, “En quête de reconnaissance. La justice spatiale à l'épreuve de l'hétéronormativité", in Justice spatiale, n 3, 2011 [en ligne]. 